\title{
Caracterización de condiciones laborales de egresados de fisioterapia de la Universidad de Boyacá, Colombia
}

\section{Characterization of working conditions of graduate physiotherapists at the University of Boyacá, Colombia}

Rocío del Pilar Castellanos-Vega ${ }^{1}$
Yolvi Amileth Rodríguez-Navas ${ }^{2}$

Recibido: noviembre 8 de 2016 Aceptado: febrero 21 de 2017

\begin{abstract}
Resumen
El objetivo de este trabajo fue el de caracterizar las condiciones socio-demográficas y los aspectos generales del ejercicio profesional de los egresados de Fisioterapia, como parte del macroproyecto: Condiciones laborales y de salud de los Fisioterapeutas en Colombia. Se trata de un estudio descriptivo, de corte transversal, con una muestra de 114 egresados del programa de fisioterapia de la Universidad de Boyacá, Colombia. La edad promedio fue de 33,3 años, sexo predominante femenino con un 95,6\%; el estado civil más frecuente fue soltero con un $47,4 \%$, mientras que el $50 \%$ de los entrevistados pertenecían al estrato social 3. Entre los aspectos profesionales se destaca que el $90,4 \%$ trabaja en empleos relacionados con la profesión, el $49 \%$ con formación en posgrado; además, el $68 \%$ trabaja entre 8 y 10 horas diarias; a pesar de las horas laboradas, se observa que el $36,9 \%$ de los egresados reciben entre 2 y 4 salarios mínimos. Lo anterior constituye un promedio salarial similar a programas como enfermería y administración en salud, lo cual evidencia la precaria remuneración de este tipo de profesionales en Colombia.
\end{abstract}

Palabras Clave: fisioterapia, condiciones de trabajo, personal de salud.

\begin{abstract}
The objective of this research work was to characterize the socio-demographic conditions and the general aspects of the professional practice of physiotherapy graduates as part of the macroproject called "Working and health conditions of physiotherapists in Colombia". It is a descriptive, cross-sectional study, using a sampling technique of 114 graduates from the Physiotherapy program at THE University of Boyacá, Colombia. The average age was 33.3 years, predominant female sex $95.6 \%$. The most frequent civil status: single $47.4 \%$, while $50 \%$ of the interviewee belonged to the social status 3. Regarding the professional aspects; currently $90.4 \%$ of physiotherapists are working in jobs related to their profession, $49 \%$ of them hold a postgraduate degree; $68 \%$ work an average of 8 and 10 hours a day. Despite the working conditions, it is observed that the hours worked, $36.9 \%$ of the graduates receive between 2 and 4 minimum Colombian wages. This average salary is comparable to that of other programs such as nursing and healthcare administration, which shows the precarious remuneration of this type of professionals in Colombia.
\end{abstract}

Keywords: physiotherapy, working conditions, health personnel. 


\section{Introducción}

Los estudios donde se realiza el seguimiento y caracterización de los egresados de diversas disciplinas, son una práctica frecuente entre las Instituciones de Educación Superior, IES, tanto nacionales como internacionales (Torres-Ortíz, \& Duarte, 2016; Fernández-Ortega, Ortíz-Montalvo, Ponce-Rosas, Fajardo-Ortíz, \& Masón-Ramírez, 2016; Cerón-Alvarez, Mesa-Laverde, \& Rojas-Morales, 2012). Este tipo de estudios tiene un gran impacto en las IES que los efectúan, porque la comunidad evalúa la calidad de la Universidad mediante las posibilidades laborales, rangos salariales, categorías de los cargos y trayectoria profesional de sus egresados (Báez-Roa, 2010; Ramirez-Leon, 2010). De esta forma la sociedad identifica la calidad en la formación recibida, la pertinencia del tipo de herramientas y métodos que el egresado porta y que puede aplicar en las organizaciones, la competitividad y el reconocimiento de la institución, lo que determina el nivel de apropiación de los productos universitarios (Argote, Duque, González, Payán, Payán, Rojas, \& Tovar, 2001; Núñez-Pérez, 2015).

En la última década se ha vuelto la mirada hacia el egresado como agente participativo en el desarrollo de las instituciones educativas y se le ha otorgado reconocimiento social (Argote et al., 2001; Manning-Bula, 2015); esto permite que el egresado sea uno de los indicadores que facilitan la medición del impacto en el medio (Cano-Gamboa, 2008), pues su empleabilidad debe ser motivo de preocupación y un compromiso de responsabilidad social. En este sentido, varios estudios han mostrado que hay una evidente relación entre la calidad de un programa académico y la empleabilidad de sus egresados (Mujica, García, Marín, \& Pérez, 2006; Naranjo-Pérez, 2008); otro ejemplo lo constituye la investigación de la Fundación Universitaria María Cano de Medellín, donde establecieron la calidad del programa por medio de la medición del impacto laboral del egresado de la carrera de fonoaudiología (Rojas-Gil, 2012). Los estudios realizados en la Universidad de Chiapas y en la Universidad Autónoma de Baja California
(Román et al., 2014; Llamas, 2014), son buenos ejemplos de cómo, a través del seguimiento de los egresados, es posible establecer la pertinencia y calidad en programas de educación superior. Se debe tener claridad en que para poder avanzar a la fase de la medición de la calidad del programa a través de los egresados, es importante identificar las condiciones laborales generales de los mismos (Hurtado-Ochoa, 2007).

En Colombia, los lineamientos expedidos por el Consejo Nacional de Acreditación, CNA, cuentan con un factor para el seguimiento y caracterización del desempeño de los egresados de los programas de educación superior (Romero-González, 2014; Lago-de Vergara, Gamoba-Suárez, \& Montes-Miranda, 2014); allí se considera que: "un programa de alta calidad se reconoce a través del desempeño laboral de sus egresados y del impacto que éstos tienen en el proyecto académico y en los procesos de desarrollo social, cultural y económico en sus respectivos entornos" (CNA, 2013). En este sentido, el programa de Fisioterapia de la Universidad de Boyacá, al ser reconocido como de alta calidad, se interesa por conocer las condiciones de trabajo de los egresados, para orientar acciones que promuevan el desarrollo y ejercicio profesional (López, Montoya, Cardona \& Bermúdez, 2000); entre ellas se encuentra la realimentación del currículo y su adecuación para asegurar una mayor pertinencia a nivel local, regional y nacional (Monagas, 2006). Sin embargo, esta intención se dificulta por la distancia existente entre los egresados y el alma mater; en Colombia es notoria la desvinculación que existe entre las instituciones de educación superior y sus egresados (Guzmán, Febles, Corredera, Flores, \& Rodríguez, 2008), realidad a la cual no es ajena la Universidad de Boyacá.

Este artículo presenta los resultados del estudio sobre las condiciones laborales de los Fisioterapeutas de la Universidad de Boyacá, Colombia, el cual hace parte del macroproyecto: condiciones laborales y de salud de los Fisioterapeutas en Colombia. El proyecto tiene como fin el de caracterizar las condiciones sociodemográficas y los as- 
pectos generales del ejercicio profesional de los egresados de Fisioterapia a nivel nacional, con el objetivo de contar con información precisa sobre las condiciones laborales de estos profesionales (Vélez-Castañeda, 2015). De esta forma será posible establecer estrategias que propendan por el mejoramiento de la calidad del ejercicio profesional de los fisioterapeutas, desde los diferentes cuerpos colegiados de esta profesión, como son: la Asociación Colombiana de Fisioterapia, ASCOFI, Asociación Colombiana de Facultades de Fisioterapia, ASCOFAFI, y el Colegio de Fisioterapia, COLFI (Gordillo, \& López, 2011).

\section{Materiales y métodos}

Se trata de un estudio descriptivo, de corte transversal (lafrancesco, 2011). La técnica de muestreo fue no probabilística por conveniencia; para la obtención de la muestra se trabajó con una $p=0,5$, nivel de confianza del $95 \%$ y una precisión del $8,2 \%$, en una población de 536 egresados, dando como resultado un tamaño de muestra de 114 egresados. Entre los criterios de selección se tienen: ser egresados del programa de Fisioterapia de la Universidad de Boyacá, UB, que diligenciaron el instrumento vía online; este cuenta con una consistencia interna, según Alfa de Cronbach, para cada dimensión, así: Valor social de la profesión de 0,793; Exposiciones riesgosas del trabajo de 0,666; Exigencias del trabajo de 0,779; Eventos en salud de 0,809; Condiciones riesgosas asociadas a la tarea de 0,905; Organización de las Tareas de 0,974 y Satisfacción con el empleo de 0,984. Según la Resolución 8430 de 1993, esta investigación se considera sin riesgo, donde se procura la confidencialidad y privacidad del individuo, desde los aspectos éticos y legales (Olivero, Domínguez, \& Malpica, 2008). Además, los participantes aceptaron el consentimiento informado.

Las variables de análisis son las sociodemográficas, los aspectos profesionales, los trabajos actuales en fisioterapia y los campos y áreas de desempeño. Las variables cuantitativas se analizaron a través del promedio, la desviación estándar y el coeficiente de variación: $>10 \%$ homogéneo, 10\%$20 \%$ medianamente homogéneo y $>20 \%$ heterogéneo; para las variables cualitativas se utilizaron frecuencias absolutas y relativas. Estas variables se analizaron para los graduados entre los años de 1997 a 2012.

\section{Resultados y discusión}

\subsection{Características sociodemográficas}

En este estudio la edad promedio fue de 33,3 $\pm 4,9$ años, con una edad mínima de 22 y una máxima de 46 años; la variabilidad fue medianamente homogénea $(C V=14,9 \%)$, aspecto que coincide con parámetros nacionales reportados en el estudio de caracterización Laboral del Talento Humano en Salud de Colombia, donde cerca del $78 \%$ de los profesionales se encuentran entre los 26 y 35 años de edad (Ministerio de Salud y Protección Social, 2012). El sexo predominante fue el femenino, con un $95,6 \%$ y en menor proporción el sexo masculino con un $4,4 \%$. El estado civil más frecuente fue el soltero en un $47,4 \%$, seguido por casado en un $33,3 \%$, unión libre en un $14 \%$ y separado en un $5,3 \%$. Respecto al estrato social se halló que el $0,9 \%$ pertenecen al estrato uno, el $17,5 \%$ al estrato dos, el $50 \%$ al estrato tres, el $23,7 \%$ al estrato cuatro y el $4,4 \%$ al estrato cinco; el restante 3,5\% registra estrato cero.

En cuanto al nivel de formación de los egresados, se identificó que el 50,9\% cuenta con formación de pregrado, el $36,8 \%$ culminó un programa de especialización y el $12,3 \%$ restante terminó estudios de maestría. Estos datos son levemente superiores a los referidos por la Universidad del Cauca, donde afirman que el $13 \%$ de los egresados del programa de Fisioterapia cuentan con estudios de posgrado finalizados y el $21 \%$ los están cursando (Chamorro, Paz, \& Vernaza, 2009). Estas son cifras interesantes si se tiene en cuenta que en el año 2010, tan solo alrededor de 96 mil profesionales colombianos accedieron a estudios posgraduales; en efecto, a medida que se incrementa el nivel posgradual disminuye el número de profesionales, teniendo para 
2010: 70.247 para Especialización, 24.309 para Maestría y 2.392 para Doctorado (Anzola, 2011), datos que corresponden con la tendencia del presente estudio. Otro trabajo es el realizado en la Pontificia Universidad Javeriana, PUJ, donde se reporta que el $17,6 \%$ de los egresados han realizado especialización, el $15 \%$ maestría y el $1 \%$ doctorado (Pérez-Alemán, 2012), datos que muestran la preferencia actual por iniciar programas de maestría, hecho que aún no se refleja en la población de este estudio, donde el mayor porcentaje de formación posgradual corresponde a especialización.

\subsection{Aspectos profesionales}

Se encontró que actualmente el 90,4\% ( $n=103)$ está trabajando en empleos relacionados con la profesión, y el 9,6\% $(n=11)$ restante, se encuentran desempeñando actividades no relacionadas con el ejercicio de la Fisioterapia; estos datos son coincidentes con el estudio de la Fundación María Cano de Medellín (Rojas, 2012). A continuación se analizan los datos correspondientes a los 103 egresados que laboran en trabajos relacionados con la fisioterapia:

En relación al tiempo cesante, entre la fecha de graduación de pregrado hasta el primer trabajo transcurrió menos de un año en el $65,8 \%$, el $22,5 \%$ entre 1 y 2 años, 6,3\% entre 3 y 4 años, 2,7\% entre 5 y 6 años, mientras que solo el $2,7 \%$ tardó más de 6 años.

En cuanto a la naturaleza jurídica de las empresas donde trabajan los egresados de Fisioterapia de la UB, priman las empresas de carácter privado con un $64,9 \%$, seguidos de la empresa pública con un $30,7 \%$ y las organizaciones sin ánimo de lucro con un $4,4 \%$.

Estos resultados son similares a los reportados en el estudio de seguimiento a recién egresados de programas académicos de pregrado de la Sede Central de la PUJ (Pérez Alemán, 2012), donde el $67,5 \%$ de los asalariados están vinculados a empresas privadas; igualmente, el $64 \%$ de los egresados del programa de enfermería de la Universidad de Caldas, laboran en empresas de carácter privado (García-Ospina, López-Ríos, Osorio-Gómez \& Realpe-Delgado, 2007); internacionalmente los resultados de esta investigación son consistentes con lo reportado por el Instituto Politécnico Nacional de México, donde aproximadamente el $70 \%$ de los egresados laboran en empresa privada (Hernández, Tavera, \& Jiménez, 2012). Sin embargo, es significativa la diferencia porcentual de los vinculados a entidades gubernamentales, $30,7 \%$ en este estudio, cuando se compara con el $11,7 \%$ de la PUK (Pérez-Alemán, 2012), lo que equivale a que un porcentaje representativo de los egresados de la Universidad de Boyacá se encuentran laborando en entidades públicas.

Respecto a las horas diarias que se trabaja por jornada se encuentran datos que superan la jornada laboral legal, pues el 32,5\% labora entre 10 y 12 horas diarias, mientras que el $40,4 \%$ labora 8 horas diarias, valor que coincide con la jornada laboral de ocho horas al día y cuarenta y ocho a la semana establecidas en Colombia (Asociación Nacional de Empresarios de Colombia, 2013). El 8,7\% trabaja por horas y el $17,5 \%$ restante labora en medio tiempo o tiempo parcial.

Es importante mencionar que una de las preocupaciones de los fisioterapeutas es el ingreso mensual recibido por el ejercicio profesional, identificándose en este estudio que el $48,5 \%$ percibe ingresos menores a dos salarios mínimos, el 36,9\% Entre 2 y 4 salarios mínimos, mientras que solo el $14,6 \%$ percibe Entre 4 y 8 salarios mínimos. Estos resultados coinciden con la tendencia nacional reportada por el Ministerio de Salud y Protección Social del año 2012, que destaca que de las profesiones analizadas: medicina, enfermería, odontología, bacteriología y fisioterapia, el Talento Humano en Salud con formación en Fisioterapia refiere el ingreso promedio más bajo, alcanzando cerca de $\$ 952.000$ (Ministerio de Salud y Protección Social, 2012); esos valores también coinciden con los resultados obtenidos por la PUJ y la Universidad del Cauca (Pérez-Alemán, 2012; Chamorro, Paz-Peña \& Vernaza-Pinzón, 2009). 
El 36,9\% de los encuestados recibe entre 2 y 4 salarios mínimos mensuales, datos equiparables a los encontrados en el estudio de condiciones laborales de las enfermeras (Cogollo, \& Gómez, 2010; Álvarez et al., 2002) y los profesionales en administración en salud de la Universidad de Antioquia (Mejía, Nieto, Arboleda, \& Montoya, 2012), lo que hace evidente la precaria situación laboral de los profesionales en salud. Estos bajos ingresos no se presentan únicamente en Colombia, pues una situación similar se detectó en un estudio realizado en América Central donde, a pesar de no encontrar problemas de desempleo, si se identifican bajos salarios, ofertas de trabajo poco atractivas y falta de estímulo salarial (Green, \& Urbina, 2004).

Se puede considerar que los ingresos recibidos están directamente conexos con la relación laboral que actualmente existe en Colombia y en particular en el sector salud, que a partir de la Ley 100 sufrió algunos cambios relacionados con la introducción al sector de nuevos modelos de vinculación laboral (Flórez, Atehortúa, \& Arenas, 2009; Avellaneda, Seidel, Londoño, \& Gonzáles, 2012). La relación laboral ha venido desmejorando notablemente: antes de la década de los 80 se prohibían contratos inferiores a 1 año; mientras que ahora, inmersos en un sistema laboral tan flexible, lo que se promueve es la desprotección de los trabajadores (Bernal, 2011).
Lo anterior es consistente con los resultados de este estudio, donde la contratación a término indefinido es del $20,4 \%$, por orden de prestación de servicios $45,6 \%$, a término fijo con un $25,2 \%$ e independiente $8,7 \%$. Este aspecto no es generalizable a todos los profesionales, puesto que dentro de la revisión de literatura se encontró que los egresados de enfermería de la Universidad de Caldas cuentan con contrato a término indefinido en un 51\% (García-Ospina, López-Ríos, Osorio-Gómez \& Realpe-Delgado, 2007), mientras que un $57 \%$ de los egresados del programa de contaduría de la Universidad Autónoma de Chiapas en México, se encuentran en esta condición (Román et al., 2014).

Es importante destacar que los fisioterapeutas de la UB, se encuentran laborando en diferentes sectores, predominando el sector salud con un $79,6 \%$, seguido por el sector educativo con un $16,5 \%$, mientras que el restante $3,9 \%$ se ubica en otros sectores.

Al realizar el análisis de la edad versus los ingresos se identificó que el $40 \%$ de los egresados mayores de 30 años reciben entre 1 y 2 salarios mínimos mensuales vigentes y tan solo el $2.4 \%$ recibe entre 6 y 8 salarios; sin embargo, se puede observar que a esta edad aumenta en un $7.7 \%$ el número de egresados que reciben entre 3 y 4 salarios mínimos, disminuyen los egresados con ingresos entre 1 y 2 salarios, pero aumentan los egresados que reciben menos de 1 salario mínimo, ver tabla 1.

\begin{tabular}{|c|c|c|c|c|c|}
\hline & & \multicolumn{4}{|c|}{ Edad } \\
\hline & & \multicolumn{2}{|c|}{$<0$ igual a 30 años } & \multicolumn{2}{|c|}{ > 30 años } \\
\hline & & Egresados & $\begin{array}{c}\text { \% del n de la } \\
\text { columna }\end{array}$ & Egresados & $\begin{array}{l}\% \text { del } n \text { de la } \\
\text { columna }\end{array}$ \\
\hline \multirow{6}{*}{ 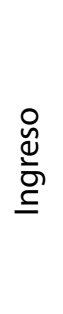 } & Entre 1 y 2 salarios & 15 & $46,9 \%$ & 33 & $40,2 \%$ \\
\hline & Entre 2 y 3 salarios & 7 & $21,9 \%$ & 17 & $20,7 \%$ \\
\hline & Entre 3 y 4 salarios & 3 & $9,4 \%$ & 14 & $17,1 \%$ \\
\hline & Entre 4 y 6 salarios & 5 & $15,6 \%$ & 9 & $11,0 \%$ \\
\hline & Entre 6 y 8 salarios & 0 & $0,0 \%$ & 2 & $2,4 \%$ \\
\hline & Menos de 1 salario & 2 & $6,2 \%$ & 7 & $8,5 \%$ \\
\hline
\end{tabular}

Tabla 1. Comparación entre edad e ingresos mensuales de los egresados de fisioterapia. 
Al realizar la comparación entre ingresos mensuales y promedio de horas diarias trabajadas, se puede observar que los fisioterapeutas que traba- jan 12 horas diarias no superan ingresos mayores a 4 salarios mínimos, ver tabla 2).

\begin{tabular}{|c|c|c|c|c|c|}
\hline & \multicolumn{5}{|c|}{$\begin{array}{c}\text { Ingresos Mensuales que Recibe Derivados de su } \\
\text { Ejercicio Profesional }\end{array}$} \\
\hline & & $\begin{array}{c}\text { Entre menos de } 1 \\
\text { salario y } 2 \text { salarios } \\
\text { mínimos }\end{array}$ & $\begin{array}{l}\text { Entre } 2 \text { y } \\
4 \text { salarios } \\
\text { mínimos }\end{array}$ & $\begin{array}{l}\text { Entre } 4 \text { y } \\
8 \text { salarios } \\
\text { mínimos }\end{array}$ & Total \\
\hline \multirow{5}{*}{$\begin{array}{l}\text { Promedio de Horas Diarias } \\
\text { que Trabaja en su Jornada }\end{array}$} & 4 horas & $100,0 \%$ & & & $100,0 \%$ \\
\hline & 6 horas & $66,7 \%$ & $16,7 \%$ & $16,7 \%$ & $100,0 \%$ \\
\hline & 8 horas & $40,0 \%$ & $42,5 \%$ & $17,5 \%$ & $100,0 \%$ \\
\hline & 10 horas & $38,7 \%$ & $45,2 \%$ & $16,1 \%$ & $100,0 \%$ \\
\hline & 12 horas & $20,0 \%$ & $80,0 \%$ & & $100,0 \%$ \\
\hline Total & & $48,5 \%$ & $36,9 \%$ & $14,6 \%$ & $100,0 \%$ \\
\hline
\end{tabular}

Tabla 2. Comparación entre horas diarias laboradas e ingresos mensuales en egresados de fisioterapia.

Al realizar la comparación entre salarios mensuales que reciben los egresados de la Universidad de Boyacá, versus el nivel de formación posgradual, se puede observar en la tabla 3 que la mayoría de quienes reciben entre 2 y 8 salarios mínimos tie- nen como máximo nivel de formación maestría; mientras que la mayoría de quienes reciben menos de 2 salarios mínimos mensuales, tan solo tienen pregrado.

\begin{tabular}{|c|c|c|c|c|c|}
\hline & & \multicolumn{3}{|c|}{$\begin{array}{c}\text { Ingresos Mensuales que Recibe Derivados de su Ejercicio } \\
\text { Profesional }\end{array}$} & \multirow[b]{2}{*}{ Total } \\
\hline & & $\begin{array}{c}\text { Entre menos de } 1 \\
\text { salario y } 2 \text { salarios } \\
\text { mínimos }\end{array}$ & $\begin{array}{l}\text { Entre } 2 \text { y } 4 \text { salarios } \\
\text { mínimos }\end{array}$ & $\begin{array}{c}\text { Entre } 4 \text { y } 8 \\
\text { salarios mínimos }\end{array}$ & \\
\hline \multirow{3}{*}{ 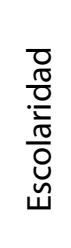 } & Pregrado & $63,5 \%$ & $30,8 \%$ & $5,8 \%$ & $100,0 \%$ \\
\hline & Especialización & $40,0 \%$ & $37,5 \%$ & $22,5 \%$ & $100,0 \%$ \\
\hline & Maestría & $9,1 \%$ & $63,6 \%$ & $27,3 \%$ & $100,0 \%$ \\
\hline Total & & $48,5 \%$ & $36,9 \%$ & $14,6 \%$ & $100,0 \%$ \\
\hline
\end{tabular}

Tabla 3. Comparación entre nivel de formación e ingresos mensuales en egresados de fisioterapia.

Adicionalmente, se supondría que a mayor y mejor formación mayor ocupación y mayor productividad, y a mayor productividad mayor salario; no obstante, en la práctica, las diferencias en los índices de ocupación, así como las diferencias en los tipos de contrato y de salarios en los diversos sectores e incluso en un mismo sector, no se explican solo por las diferencias en la formación, que en 
muchos casos no influyen en el salario asignado (Ávila, \& Aguirre, 2005; de Vries, Vázquez-Cabrera, \& Rios-Treto, 2013; García-Rubiano, \& Forero-Aponte, 2015). Esta situación explica de cierta forma por qué los profesionales de fisioterapia no continúan con formación posgradual, especialmente programas de maestría y doctorado.

\section{Conclusiones}

Como resultado de este estudio se identificó que la edad promedio de los egresados del Programa de Fisioterapia de la Universidad de Boyacá, es 33 años, en su mayoría de sexo femenino y estrato 3 , datos que coinciden con los resultados de la caracterización laboral del talento humano en salud realizada por el Ministerio de Salud y Protección Social.

En cuanto a la formación posgradual el 49\% cuenta con este tipo de formación, ya sea especialización y/o maestría, datos que coinciden con los resultados de estudios realizados en Colombia, en instituciones como la Pontificia Universidad Javeriana y la Universidad del Cauca.

Del total de los egresados participantes en el estudio, un $90 \%$ se desempeñan en trabajos relacionados con la profesión, en su mayoría (65\%) en empresa privada con un promedio de horas diarias laboradas de 8 , y remuneración que oscila entre 1 y 4 salarios mínimos mensuales vigentes, constituyéndose en un promedio salarial similar a carreras como enfermería y administración en salud, hecho que hace evidente la precaria remuneración salarial de los profesionales de ciencias de la salud.

En general, se puede concluir que la caracterización de los egresados del Programa de Fisioterapia de la Universidad de Boyacá, coinciden con los obtenidos en otros estudios realizados a nivel nacional.

\section{Referencias}

Álvarez, C., Campos, L., Cerón, G., Franco, B., Ledesma, M., Mora, I. A. et al. (2002). Mercado laboral de enfermería en la ciudad de Guanajuato. Acta Universitaria, 12 (2), 20 - 27.

Anzola, G. (2011). Realidad de los posgrados en Colombia y su situación frente a la reforma de la Ley 30. Revista UDCA Actualidad \& Divulgación Científica, 14 (2), 3-5. Recuperado de: http://www. scielo.org.co/scielo.php?script=sci_arttext\&pi$d=S 0123-42262011000200001$

Argote, L. Á., Duque, Á. M., González, L., Payán, A., Payán, C. M., Rojas, L. H., \& Tovar, M. C. (2001). Los egresados y su desempeño en el medio: un desafío de las instituciones formadoras del recurso humano en salud. Colombia Médica, 32 (4), 169-173. Recuperado de: http://www.redalyc.org/articulo. oa?id=28332403

Asociación Colombiana de Fisioterapia., \& SENA. (2008). Caracterización de la Profesión de Fisioterapia en Colombia. Bogotá. Recuperado de: http://observatorio.sena.edu.co/mesas/01/SERVICIOS\%20A\%20LA\%20SALUD\%20FISIOTERAPIA. pdf

Asociación Nacional de Empresarios de Colombia. ANDI. (2013). Contratación: Marco Legal Laboral y de la Seguridad Social 2013. Jornada Laboral. Recuperado de: http://www.andi.org.co/es/GAI/GuiInv/VisEst/MLSS/Paginas/JorLab.aspx

Avellaneda, C., Seidel, A., Londoño, A., \& Gonzáles, C. (2012). Caracterización socioeconómica, geográfica y laboral de los médicos dermatólogos en Colombia. Revista de la Asociación Colombiana de Dermatología \& Cirugía Dermatológica, 20 (2), 129134. 
Ávila, M., \& Aguirre, C. (2005). El seguimiento de los egresados como indicador de la calidad docente. Revista Electrónica Interuniversitaria de Formación del Profesorado, 8 (3), 1-5. Recuperado de: http:// redalyc.org/articulo.oa?id $=217017156006$

Báez-Roa, M. (2010). Seguimiento a egresados de la Escuela de Administración Industrial de la Universidad Pedagógica y Tecnológica de Colombia. Revista de Investigación, Desarrollo e Innovación, 1 (1), 30-39. Recuperado de: http://revistas.uptc. edu.co/revistas/index.php/investigacion_duitama/article/view/1291

Bernal, F. (2011). Panorama de la contratación laboral médica en Colombia. Revista Médico Legal, 17 (1), 6-9. Recuperado de: http://www.medicolegal.com.co/pdf/esp/2011/1/Bajas\%20RML\%20 Mayo\%20-\%20Septiembre/Aspectos\%20Laborales.pdf

Cano, C. (2008). Determinantes en la eficiencia en la producción de educación. Revista de la Educación Superior, 37 (147), 17-30. Recuperado de: http://www.scielo.org.mx/pdf/resu/v37n147/ v37n147a2.pdf

Cerón-Álvarez, D. C., Mesa-Laverde, Y. C., \& Rojas-Morales, C. E. (2012). La naturaleza del conocimiento matemático y su impacto en las concepciones del profesor. Revista de Investigación, Desarrollo e Innovación, 2 (2), 49-59. Recuperado de: http://revistas.uptc.edu.co/index.php/investigacion_duitama/article/view/1316

Chamorro, A. P., Paz, C. I., \& Vernaza, P. (2009). Características laborales de los egresados del programa de Fisioterapia de la Universidad del Cauca e impacto sobre el medio 2006- 2008. Revista Facultad de Ciencias de la Salud, 11 (4), 9-20.

Cogollo, Z. \& Gómez, E. (2010). Condiciones laborales en enfermeras de Cartagena, Colombia. Avances en Enfermería, 28 (1), 31-38.
Consejo Nacional de Acreditación. (2013). Lineamientos para la acreditación de los programas de pregrado. Bogotá: CNA.

De Vries, W., Vázquez, R., \& Rios, D. (2013). Millonarios o malparados: ¿de qué depende el éxito de los egresados universitarios? Revista lberoamericana de Educación Superior, 4 (9), 3-20. Recuperado de: http://www.redalyc.org/articulo. oa?id=299126789001

Fernández-Ortega, M. A., Ortíz-Montalvo, A., Ponce-Rosas, E. R., Fajardo-Ortíz, G., \& Masón-Ramírez, J. J. (2016). Caracterización de alumnos de la carrera de medicina. Investigación en Educación Médica, 5 (19), 148-154.

Flórez, J., Atehortua, S., \& Arenas A. (2009). Las condiciones laborales de los profesionales de la salud a partir de la Ley 100 de 1993: evolución y un estudio de caso para Medellín. Revista Gerencia y Políticas de Salud, 8 (16), 107-131.

García, C., López, L. H., Osorio, L. S., \& Realpe, C. (2007). Desempeño profesional de los egresados del programa de enfermería de la Universidad de Caldas y su relación con la ley de seguridad social en salud y con el perfil de formación (Manizales 1995-2004). Revista Hacia la Promoción de la Salud, 12, 91-108. Recuperado de: http://www.redalyc. org/articulo.oa?id=309126689008

García-Rubiano, M., \& Forero-Aponte, C. (2015). Contrato psicológico y cambio organizacional en una entidad perteneciente al sector terciario de la ciudad de Bogotá, Colombia. Revista de Investigación, Desarrollo e Innovación, 6 (1), 15-28. doi: http://doi.org/10.19053/20278306.4047

Gordillo, E., \& López, P. (2011). Caracterización profesional del fisioterapeuta en unidad de cuidado crítico en Bogotá. Revista Movimiento Científico, 5 (1), 25-40. 
Green, I., \& Urbina, O. (2004). Los estudios de seguimiento a egresados en América central: un esfuerzo para mejorar la relevancia de los programas de estudio en las Universidades. Revista Paradigma. 13 (17), 37 - 49.

Guzmán, S., Febles, M., Corredera, A., Flores, P., \& Rodríguez, P. (2008). Estudio de seguimiento de egresados: recomendaciones para su desarroIlo. Innovación Educativa, 8 (42), 19-31. Recuperado de: http://estudiosterritoriales.org/articulo. oa?id=179421234003

Hernández, C., Tavera, M., \& Jiménez, M. (2012). Seguimiento de Egresados en Tres Programas de Maestría en una Escuela del Instituto Politécnico Nacional en México. Formación Universitaria, 5 (2), 41-52. doi: https://dx.doi.org/10.4067/S071850062012000200006

Hurtado, C. (2007). Impacto Social de los Programas de Pregrado del programa de economía en la Universidad de EAFIT. Recuperado de: http:// www.graduadoscolombia.edu.co/html/1732/articles-155955_archivo_pdf.unknown

lafrancesco, G. M. (2011). Algunas problemáticas de la investigación y pedagogía en educación en Colombia: estrategias para enfrentarlas y resolverlas. Revista de Investigación, Desarrollo e Innovación, 1 (2), 7-16. Recuperado de: http://revistas. uptc.edu.co/index.php/investigacion_duitama/ article/view/1298

Lago-de Vergara, D., Gamoba-Suárez, A. A., \& Montes-Miranda, A. J. (2014). Calidad de la educación superior: un análisis de sus principales determinantes. Saber, Ciencia y Libertad, 9 (1), 157-170. DOI: http://dx.doi.org/10.22525/sabcliber.2014v9n1.157170

Llamas, G. (2014). Pertinencia y calidad en Programas de estudios superiores. Global Conference on Business and Finance Proceedings, 9 (2), 1523-1529.
Manning-Bula, L. (2015). Impacto de los egresados de los programas técnicos laborales. Una panorámica nacional e internacional del estado del arte. Saber, Ciencia Y Libertad, 10 (1), 189-202. doi:http://dx.doi.org/10.22525/sabcliber.2015v10n1.189202

Mejía, L., Nieto, E., Arboleda, G., \& Montoya, M. (2012). Perfil socio-laboral de los profesionales en Administración en Salud de la Universidad de Antioquia 1999-2008. Revista Facultad Nacional de Salud Pública, 30 (2), 152-162.

Ministerio de Salud y Protección Social. (2012). Caracterización laboral del talento humano en salud de Colombia: aproximaciones desde el ingreso base de cotización al sistema general de seguridad social en salud. Recuperado de: http://www.minsalud.gov.co/salud/Documents/Observatorio $\% 20$ Talento\%20Humano\%20en\%20Salud/Caracterizaci\%C3\%B3nLaboralTHS.pdf

Monagas, D. (2006). La calidad del egresado: aspecto vital de la evaluación institucional. Actualidad Contable Faces, 9 (13), 68-77.

Mujica, M., García, R., Marín, F., \& Pérez, E. (2006). Prácticas profesionales docentes y perfil académico de egreso: estrategia para contribuir al desarrollo productivo regional. Multiciencias, 6 (2), 162-167. Recuperado de: http://www.redalyc.org/ articulo.oa?id=90460209

Naranjo-Pérez, F. (2008). La empleabilidad de los egresados es un asunto de responsabilidad social. El Hombre y la Máquina, 30, 4-7. Recuperado de: http://www.redalyc.org/articulo. oa?id=47803001

Nieto, E., Montoya, G., Cardona, E., \& Bermúdez, L. (2000). Perfil sociodemográfico y laboral de los odontólogos en la ciudad de Medellín. Revista Facultad Nacional Salud Pública, 18 (2), 41 - 53. 
Núñez-Pérez, V. (2015). Pedagogía social e interculturalismo: una lectura posible. Revista de Investigación, Desarrollo e Innovación, 5 (2), 141-149. doi: $10.19053 / 20278306.3716$

Olivero, R., Domínguez, A., \& Malpica, C. C. (2008). Principios Bioéticos Aplicados a la Investigación Epidemiológica. Acta bioéthica, 14 (1), 90-96. DOI: https://dx.doi.org/10.4067/S1726569X2008000100012

Pérez, Y. O. (Ed). (2012). Estudio de seguimiento a recién egresados de programas académicos de pregrado de la Sede Central 2012. Bogotá, Colombia: Pontificia Universidad Javeriana. Recuperado de: http://puj-portal.javeriana.edu.co/portal/page/ portal/PORTAL_VERSION_2009_2010/resources_ v4/SPEGRE2012V1.pdf

Ramirez-Leon, C. (2010). Caracterización del entorno de la Escuela de Diseño Industrial de la Uptc: una herramienta de competitividad. Revista de Investigación, Desarrollo e Innovación, 1 (1), 19-29. Recuperado de: http://revistas.uptc.edu.co/revistas/index.php/investigacion_duitama/article/ view/1290

Rojas, A. (2012). Impacto laboral del egresado del programa de fonoaudiología en la Fundación Uni- versitaria María Cano, Medellín. Revista Árete, 12 (1), 102-110

Román, J., Franco, R., \& Gordillo, A. (2014). Pertinencia educativa, elementos para su evaluación a partir de la incursión laboral de egresados. Global Conference on Business and Finance Proceedings, 9 (2), 1019-1029.

Romero-González, Z. (2014). El sistema de aseguramiento de la calidad en la educación superior. Saber, Ciencia y Libertad, 9 (2), 11-14. Recuperado de: http://sabercienciaylibertad.com/ojs/index.php/ scyl/article/view/2/0

Torres-Ortiz, J. A., \& Duarte, J. E. (2016). Los procesos pedagógicos administrativos y los aspectos socio-culturales de inclusión y tecno-pedagogía a través de las tendencias pedagógicas en educación a distancia y virtual. Revista de Investigación, Desarrollo e Innovación, 6 (2), 179-190. doi: http:// doi.org/10.19053/20278306.4606

Vélez-Castañeda, L. A. (2015). Estudio nacional sobre condiciones de trabajo y salud de los fisioterapeutas en Colombia. (Tesis de pregrado), Facultad de fisioterapia, Universidad CES. Recuperado de: http://hdl.handle.net/10946/4076 